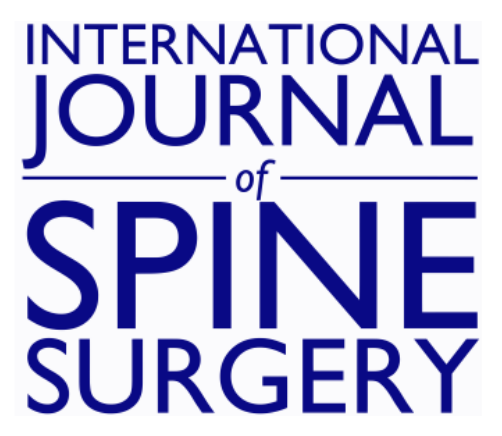

\title{
Features of Spinal Metastases: A Retrospective View
}

\author{
Koray Basdelioglu \\ Int J Spine Surg 2021, 15 (1) 119-129 \\ doi: https://doi.org/10.14444/8016 \\ http://ijssurgery.com/content/15/1/119 \\ This information is current as of April 26, 2023.
}

Email Alerts Receive free email-alerts when new articles cite this article. Sign up at: http://ijssurgery.com/alerts 


\title{
Features of Spinal Metastases: A Retrospective View
}

\author{
KORAY BAŞDELIOĞLU, MD \\ Istanbul Oncology Hospital Department of Orthopaedic and Traumatology, Istanbul, Turkey
}

\begin{abstract}
Backround: The present study aimed to determine the frequency of spinal metastases, to evaluate the features of spinal metastases, and to reveal clues to shed light on the origin of spinal metastases with unknown primary.

Methods: The data of patients who were followed up with the diagnosis of cancer in Istanbul Oncology Hospital between 2017 and 2019 were analyzed retrospectively. A total of 156 patients with spinal metastases and without visceral metastases were included in the study by applying inclusion and exclusion criteria. Clinical data, pathological diagnostic reports, and positron emission tomography-computed tomography results of 156 patients were evaluated. The groups were evaluated in terms of age, gender, number of spinal metastases (single focus, multiple focus), and localization of spinal metastasis. The spinal localization evaluation included both the main anatomical localizations and a detailed evaluation of each spine.

Results: The most common metastasis region was the thoracic spine in respiratory system cancers $(28.38 \%)$, the thoracic + lumbar spine in breast $(42.42 \%)$, prostate $(50.00 \%)$, and gynecologic $(40.00 \%)$ cancers, and the lumbar spine in gastrointestinal $(37.50 \%)$ and urinary $(30.00 \%)$ tract cancers $(P=.313)$. C5 spinal metastasis percentages were significantly higher in breast and gastrointestinal tract cancers than the others $(P=.042)$. T5 spinal metastasis percentage was significantly higher in gynecologic tumors than in the other cancers $(P=.002)$. T10 spinal metastasis percentages were significantly higher in prostate and gynecologic tumors than the others $(P=.016)$. L1 spinal metastasis percentage was significantly higher in breast tumors $(P=.009)$. L2 spinal metastasis percentages were significantly higher in breast, prostate, and gynecologic tumors $(P=.011)$. L4 spinal metastasis percentages were significantly higher in breast and prostate tumors $(P=.041)$. L5 spinal metastasis percentage was significantly higher in prostate tumors $(P$ $=.029)$ than the other cancers.

Conclusions: It was observed that primary cancers were often prone to metastasis to nearby spine. The results obtained by detailed examination of spinal metastases may provide a clinical benefit by providing clues in investigation of primary unknown cancers.
\end{abstract}

Level of Evidence: 3.

Tumor

Keywords: spine, bone, metastases, cancer, unknown

\section{INTRODUCTION}

The skeleton is the most common region where primary cancers metastasize. ${ }^{1}$ Bone metastasis is more common than primary bone cancers, especially in adults. ${ }^{2}$ Although the overall incidence of bone metastases is unknown, publications show bone metastasis occurs in two-thirds of primary cancer patients. $^{3,4}$ Breast, prostate, and lung cancers are responsible for $80 \%$ of bone metastasis due to their high prevalence. ${ }^{5,6}$

The spine is the most common site of bone metastasis. $^{7-9}$ About $70 \%$ of patients with cancer may develop spinal metastasis, and $20 \%$ of them often suffer from neurological deficits. ${ }^{10,11}$ In these patients, $10 \%$ of spinal cord compression can develop, and surgical decompression may be needed with or without stabilization. ${ }^{10,12}$ Bone metastases are the main cause of morbidity. They can cause severe pain, especially at night, decreased mobility, pathological fractures, spinal cord compression, bone marrow aplasia, and hypercalcemia. ${ }^{13-15}$

Primary cancer-to-bone metastasis occurs through systemic blood circulation or vertebral venous plexus. ${ }^{7-9}$ While overall bone metastases, especially extremity and visceral metastases, occur by bloodstream spread, diffusion from the venous system is the main process of spinal metastases. ${ }^{16-18}$ The vertebral venous plexus is a valveless network system of veins extending from the cranium to the pelvis. ${ }^{16}$ Many studies exist on the effects of systemic blood circulation on bone metastasis, but few studies have examined the effects of the vertebral venous plexus on bone metastasis. ${ }^{16,18,19}$ 
This study, in which visceral and extremity metastases occuring through blood circulation were not included, was carried out both to evaluate the features of spinal metastases and to reveal clues to shed light on the origin of spinal metastases with unknown primary.

\section{MATERIAL AND METHODS}

\section{Patient Selection and Evaluation}

The data of patients who were followed up with the diagnosis of cancer in Istanbul Oncology Hospital between 2017 and 2019 were analyzed retrospectively. Performance of 18 F-fluoro-2-deoxyglucose (FDG) positron emission tomographycomputed tomography (PET-CT), diagnosis of spinal metastasis, and complete data and contact information were determined as inclusion criteria. Exclusion criteria were determined as the presence of more than 1 primary cancer, primary bone cancer, or visceral or bone metastasis, except spinal metastasis. With the application of inclusion and exclusion criteria, 156 patients were included in the study.

The clinical data of 156 patients were analyzed retrospectively, including demographic characteristics (age, gender), pathological diagnostic reports, and PET-CT results. Primary cancers were divided into 6 groups as respiratory system cancers (lung cancer, nasopharyngeal cancer), breast cancer, gastrointestinal tract cancers (gastric cancer, pancreatic cancer, colon cancer, rectum cancer), urinary tract cancers (renal cancer, bladder cancer), prostate cancer, and gynecological cancers (endometrial cancer, cervical cancer, vagina cancer; Table 1). The groups were evaluated in terms of age, gender, number of spinal metastases (single focus, multiple focus), and localization of spinal metastasis. Spinal metastasis localization was categorized as cervical, thoracic, lumbar, cervical + thoracic, cervical + lumbar, or thoracic + lumbar + lumbar spine. In addition, the metastasis involvement features were analyzed by anatomically detailing the spine in each main spinal column (cervical, thoracic, lumbar) as corpus, posterior, and corpus + posterior. PET-CT results of 156 patients were analyzed for distribution and pattern of spinal metastases.

The study protocol was approved by Yeditepe University Ethics Committee (1227/21.05.2020). The study was carried out in accordance with the principles of the Helsinki Declaration.

\section{PET-CT Imaging and Evaluation}

All patients underwent FDG injection after 4 to 6 hours of fasting. PET-CT examination was performed 1 hour after FDG injection. A General Electric Discovery IQ Gen 2 PET-CT device (New York, NY) was used for the PET-CT of all patients. FDG uptake of lesions was evaluated as maximum standardized uptake value (SUVmax) in the images evaluated by 2 different nuclear medicine doctors who did not know the clinical features of the patients. Increased standardized uptake value (SUV), osteoblastic, osteolytic, and mixed osteoblastic and osteolytic lesions were evaluated as metastases.

\section{Statistical Analysis}

All analyses were performed on SPSS v21 (SPSS Inc., Chicago, IL). For the normality check, the Kolmogorov-Smirnov test was used. Data are given as mean \pm standard deviation for continuous variables and frequency (percentage) for categorical variables. Normally distributed variables were analyzed with independent samples $t$ tests or 1way analyses of variance (ANOVAs) depending upon count of groups. Pairwise comparisons were performed with the Tamhane test. Categorical variables were evaluated by using $\chi^{2}$ tests or Fisher's exact tests. Values of $P<.05$ were accepted as statistically significant results.

\section{RESULTS}

We included 156 patients (59 females and 97 males) in our study. Mean age was $65.21 \pm 12.19$ (range, 35-96). Males were significantly older than the females $(P=.008$; Table 1$)$. The most common primary cancer was respiratory system cancers (47.44\%) among all patients. The most common primary cancers were breast $(54.24 \%)$, respiratory system $(23.73 \%)$, and gastrointestinal tract $(10.17 \%)$ cancers in females, and respiratory system $(61.86 \%)$, prostate $(18.56 \%)$, and gastrointestinal tract $(10.31 \%)$ cancers in males $(P<.001)$. Seventyone $(73.20 \%)$ male patients had multiple metastases, while $45(76.27 \%)$ female patients had multiple metastases $(P=.812$; Table 1$)$. The most common metastasis region was thoracic + lumbar spine for both genders $(P=.292$; Table 1$)$.

Fifty-three $(33.97 \%)$ patients had cervical spinal metastases. Metastasis was most common on C5 $(15.38 \%)$ among all patients. Ten $(6.41 \%)$ of them 
Table 1. Summary of patients and metastasis characteristics regarding gender. ${ }^{\text {a }}$

\begin{tabular}{|c|c|c|c|c|}
\hline & Female $(n=59)$ & Male $(\mathbf{n}=97)$ & Total (156) & $P$ \\
\hline Age & $61.92 \pm 13.00$ & $67.22 \pm 11.28$ & $65.21 \pm 12.19$ & .008 \\
\hline \multicolumn{5}{|l|}{ Primary tumor } \\
\hline Respiratory system & $14(23.73)$ & $60(61.86)$ & $74(47.44)$ & $<.001$ \\
\hline Lung & $14(23.73)$ & $59(60.82)$ & $73(46.79)$ & \\
\hline Nasopharyngeal & $0(0.00)$ & $1(1.03)$ & $1(0.64)$ & \\
\hline Breast & $32(54.24)$ & $1(1.03)$ & $33(21.15)$ & \\
\hline Gastrointestinal tract & $6(10.17)$ & $10(10.31)$ & $16(10.26)$ & \\
\hline Gastric & $3(5.08)$ & $4(4.12)$ & 7 (4.49) & \\
\hline Pancreatic & $1(1.69)$ & $1(1.03)$ & $2(1.28)$ & \\
\hline Colonic & $2(3.39)$ & $3(3.09)$ & $5(3.21)$ & \\
\hline Rectal & $0(0.00)$ & $2(2.06)$ & $2(1.28)$ & \\
\hline Urinary tract & $2(3.39)$ & $8(8.25)$ & $10(6.41)$ & \\
\hline Renal & $2(3.39)$ & $3(3.09)$ & $5(3.21)$ & \\
\hline Urinary bladder & $0(0.00)$ & $5(5.15)$ & $5(3.21)$ & \\
\hline Prostate & $0(0.00)$ & $18(18.56)$ & $18(11.54)$ & \\
\hline Gynecologic & $5(8.47)$ & $0(0.00)$ & $5(3.21)$ & \\
\hline Endometrial & $1(1.69)$ & $0(0.00)$ & $1(0.64)$ & \\
\hline Cervical & $3(5.08)$ & $0(0.00)$ & $3(1.92)$ & \\
\hline Vaginal & $1(1.69)$ & $0(0.00)$ & $1(0.64)$ & \\
\hline \multicolumn{5}{|l|}{ Metastasis status } \\
\hline Single & $14(23.73)$ & $26(26.80)$ & $40(25.64)$ & .812 \\
\hline Multiple & $45(76.27)$ & $71(73.20)$ & $116(74.36)$ & \\
\hline \multicolumn{5}{|l|}{ Region } \\
\hline Cervical & $2(3.39)$ & $2(2.06)$ & $4(2.56)$ & .292 \\
\hline Thoracic & $9(15.25)$ & $22(22.68)$ & $31(19.87)$ & \\
\hline Lumbar & $8(13.56)$ & 19 (19.59) & $27(17.31)$ & \\
\hline Cervical + thoracic & $2(3.39)$ & $10(10.31)$ & $12(7.69)$ & \\
\hline Cervical + lumbar & $3(5.08)$ & $2(2.06)$ & $5(3.21)$ & \\
\hline Thoracic + lumbar & $20(33.90)$ & $25(25.77)$ & $45(28.85)$ & \\
\hline Cervical + thoracic + lumbar & $15(25.42)$ & $17(17.53)$ & $32(20.51)$ & \\
\hline Cervical spine & $22(37.29)$ & $31(31.96)$ & $53(33.97)$ & .612 \\
\hline $\mathrm{C} 1$ & $5(8.47)$ & $3(3.09)$ & $8(5.13)$ & .156 \\
\hline Corpus & $2(3.39)$ & $2(2.06)$ & $4(2.56)$ & \\
\hline Posterior & 1 (1.69) & $0(0.00)$ & $1(0.64)$ & \\
\hline Corpus + posterior & $2(3.39)$ & $1(1.03)$ & $3(1.92)$ & \\
\hline $\mathrm{C} 2$ & $4(6.78)$ & $8(8.25)$ & $12(7.69)$ & 1.000 \\
\hline Corpus & $2(3.39)$ & $5(5.15)$ & 7 (4.49) & \\
\hline Posterior & $0(0.00)$ & $0(0.00)$ & $0(0.00)$ & \\
\hline Corpus + posterior & $2(3.39)$ & $3(3.09)$ & $5(3.21)$ & \\
\hline $\mathrm{C} 3$ & $11(18.64)$ & $8(8.25)$ & $19(12.18)$ & .076 \\
\hline Corpus & $3(5.08)$ & $3(3.09)$ & $6(3.85)$ & \\
\hline Posterior & $1(1.69)$ & $1(1.03)$ & $2(1.28)$ & \\
\hline Corpus + posterior & $7(11.86)$ & $4(4.12)$ & $11(7.05)$ & \\
\hline $\mathrm{C} 4$ & $7(11.86)$ & $9(9.28)$ & $16(10.26)$ & .807 \\
\hline Corpus & $1(1.69)$ & $6(6.19)$ & $7(4.49)$ & \\
\hline Posterior & $1(1.69)$ & $1(1.03)$ & $2(1.28)$ & \\
\hline Corpus + posterior & $5(8.47)$ & $2(2.06)$ & $7(4.49)$ & \\
\hline $\mathrm{C} 5$ & $14(23.73)$ & $10(10.31)$ & $24(15.38)$ & .043 \\
\hline Corpus & $3(5.08)$ & $7(7.22)$ & $10(6.41)$ & \\
\hline Posterior & $3(5.08)$ & $1(1.03)$ & $4(2.56)$ & \\
\hline Corpus + posterior & $8(13.56)$ & $2(2.06)$ & $10(6.41)$ & \\
\hline C6 & $7(11.86)$ & $8(8.25)$ & $15(9.62)$ & .643 \\
\hline Corpus & $3(5.08)$ & $5(5.15)$ & $8(5.13)$ & \\
\hline Posterior & $2(3.39)$ & $1(1.03)$ & $3(1.92)$ & \\
\hline Corpus + posterior & $2(3.39)$ & $2(2.06)$ & $4(2.56)$ & \\
\hline $\mathrm{C} 7$ & $4(6.78)$ & $15(15.46)$ & $19(12.18)$ & .175 \\
\hline Corpus & $1(1.69)$ & $6(6.19)$ & 7 (4.49) & \\
\hline Posterior & $0(0.00)$ & $6(6.19)$ & $6(3.85)$ & \\
\hline Corpus + posterior & $3(5.08)$ & $3(3.09)$ & $6(3.85)$ & \\
\hline $\mathrm{C} 8$ & 1 (1.69) & $2(2.06)$ & $3(1.92)$ & 1.000 \\
\hline Corpus & $0(0.00)$ & $2(2.06)$ & $2(1.28)$ & \\
\hline Posterior & $0(0.00)$ & $0(0.00)$ & $0(0.00)$ & \\
\hline Corpus + posterior & $1(1.69)$ & $0(0.00)$ & $1(0.64)$ & \\
\hline Thoracic spine & 46 (77.97) & $74(76.29)$ & $120(76.92)$ & .964 \\
\hline $\mathrm{T} 1$ & $9(15.25)$ & $15(15.46)$ & $24(15.38)$ & 1.000 \\
\hline Corpus & $5(8.47)$ & $10(10.31)$ & $15(9.62)$ & \\
\hline Posterior & $0(0.00)$ & $3(3.09)$ & $3(1.92)$ & \\
\hline Corpus + posterior & $4(6.78)$ & $2(2.06)$ & $6(3.85)$ & \\
\hline
\end{tabular}


Table 1. Continued.

\begin{tabular}{|c|c|c|c|c|}
\hline & Female $(n=59)$ & Male $(n=97)$ & Total (156) & $P$ \\
\hline $\mathrm{T} 2$ & $12(20.34)$ & $13(13.4)$ & $25(16.03)$ & .357 \\
\hline Corpus & $7(11.86)$ & $8(8.25)$ & $15(9.62)$ & \\
\hline Posterior & $2(3.39)$ & $2(2.06)$ & $4(2.56)$ & \\
\hline Corpus + posterior & $3(5.08)$ & $3(3.09)$ & $6(3.85)$ & \\
\hline $\mathrm{T} 3$ & $12(20.34)$ & $20(20.62)$ & $32(20.51)$ & 1.000 \\
\hline Corpus & $6(10.17)$ & $12(12.37)$ & $18(11.54)$ & \\
\hline Posterior & $1(1.69)$ & $0(0.00)$ & $1(0.64)$ & \\
\hline Corpus + posterior & $5(8.47)$ & $8(8.25)$ & $13(8.33)$ & \\
\hline $\mathrm{T} 4$ & $11(18.64)$ & $15(15.46)$ & $26(16.67)$ & .768 \\
\hline Corpus & $6(10.17)$ & $11(11.34)$ & $17(10.9)$ & \\
\hline Posterior & $3(5.08)$ & $1(1.03)$ & $4(2.56)$ & \\
\hline Corpus + posterior & $2(3.39)$ & $3(3.09)$ & $5(3.21)$ & \\
\hline T5 & $15(25.42)$ & $12(12.37)$ & $27(17.31)$ & .061 \\
\hline Corpus & $8(13.56)$ & $7(7.22)$ & $15(9.62)$ & \\
\hline Posterior & $2(3.39)$ & $1(1.03)$ & $3(1.92)$ & \\
\hline Corpus + posterior & $5(8.47)$ & $4(4.12)$ & $9(5.77)$ & \\
\hline T6 & $14(23.73)$ & $17(17.53)$ & $31(19.87)$ & .463 \\
\hline Corpus & $11(18.64)$ & $10(10.31)$ & $21(13.46)$ & \\
\hline Posterior & $1(1.69)$ & $0(0.00)$ & $1(0.64)$ & \\
\hline Corpus + posterior & $2(3.39)$ & $7(7.22)$ & $9(5.77)$ & \\
\hline $\mathrm{T} 7$ & $18(30.51)$ & $21(21.65)$ & $39(25.00)$ & .294 \\
\hline Corpus & $15(25.42)$ & $14(14.43)$ & $29(18.59)$ & \\
\hline Posterior & $0(0.00)$ & $1(1.03)$ & $1(0.64)$ & \\
\hline Corpus + posterior & $3(5.08)$ & $6(6.19)$ & $9(5.77)$ & \\
\hline $\mathrm{T} 8$ & $21(35.59)$ & $18(18.56)$ & $39(25.00)$ & .028 \\
\hline Corpus & $16(27.12)$ & $9(9.28)$ & $25(16.03)$ & \\
\hline Posterior & $2(3.39)$ & $0(0.00)$ & $2(1.28)$ & \\
\hline Corpus + posterior & $3(5.08)$ & $9(9.28)$ & $12(7.69)$ & \\
\hline T9 & $14(23.73)$ & $26(26.80)$ & $40(25.64)$ & .812 \\
\hline Corpus & $13(22.03)$ & $16(16.49)$ & $29(18.59)$ & \\
\hline Posterior & $0(0.00)$ & $3(3.09)$ & $3(1.92)$ & \\
\hline Corpus + posterior & $1(1.69)$ & $7(7.22)$ & $8(5.13)$ & \\
\hline $\mathrm{T} 10$ & $19(32.20)$ & $25(25.77)$ & $44(28.21)$ & .495 \\
\hline Corpus & $15(25.42)$ & $11(11.34)$ & $26(16.67)$ & \\
\hline Posterior & $0(0.00)$ & $4(4.12)$ & $4(2.56)$ & \\
\hline Corpus + posterior & $4(6.78)$ & $10(10.31)$ & $14(8.97)$ & \\
\hline $\mathrm{T} 11$ & $14(23.73)$ & $25(25.77)$ & $39(25.00)$ & .924 \\
\hline Corpus & $8(13.56)$ & $14(14.43)$ & $22(14.10)$ & \\
\hline Posterior & $2(3.39)$ & $1(1.03)$ & $3(1.92)$ & \\
\hline Corpus + posterior & $4(6.78)$ & $10(10.31)$ & $14(8.97)$ & \\
\hline $\mathrm{T} 12$ & $20(33.90)$ & $23(23.71)$ & $43(27.56)$ & .232 \\
\hline Corpus & $8(13.56)$ & $8(8.25)$ & $16(10.26)$ & \\
\hline Posterior & $1(1.69)$ & $2(2.06)$ & $3(1.92)$ & \\
\hline Corpus + posterior & $11(18.64)$ & $13(13.40)$ & $24(15.38)$ & \\
\hline Lumbar vertebrae & $46(77.97)$ & $63(64.95)$ & $109(69.87)$ & .124 \\
\hline $\mathrm{L} 1$ & $25(42.37)$ & $21(21.65)$ & $46(29.49)$ & .010 \\
\hline Corpus & $11(18.64)$ & $10(10.31)$ & $21(13.46)$ & \\
\hline Posterior & $4(6.78)$ & $3(3.09)$ & $7(4.49)$ & \\
\hline Corpus + posterior & $10(16.95)$ & $8(8.25)$ & $18(11.54)$ & \\
\hline $\mathrm{L} 2$ & $26(44.07)$ & $28(28.87)$ & $54(34.62)$ & .078 \\
\hline Corpus & $12(20.34)$ & $11(11.34)$ & $23(14.74)$ & \\
\hline Posterior & $3(5.08)$ & $4(4.12)$ & $7(4.49)$ & \\
\hline Corpus + posterior & $11(18.64)$ & $13(13.40)$ & $24(15.38)$ & \\
\hline L3 & $27(45.76)$ & $33(34.02)$ & $60(38.46)$ & .196 \\
\hline Corpus & $11(18.64)$ & $18(18.56)$ & $29(18.59)$ & \\
\hline Posterior & $4(6.78)$ & $4(4.12)$ & $8(5.13)$ & \\
\hline Corpus + posterior & $12(20.34)$ & $11(11.34)$ & $23(14.74)$ & \\
\hline L4 & $23(38.98)$ & $30(30.93)$ & $53(33.97)$ & .392 \\
\hline Corpus & $9(15.25)$ & $15(15.46)$ & $24(15.38)$ & \\
\hline Posterior & $1(1.69)$ & $3(3.09)$ & $4(2.56)$ & \\
\hline Corpus + posterior & $13(22.03)$ & $12(12.37)$ & $25(16.03)$ & \\
\hline $\mathrm{L} 5$ & $21(35.59)$ & $32(32.99)$ & $53(33.97)$ & .874 \\
\hline Corpus & $10(16.95)$ & $15(15.46)$ & $25(16.03)$ & \\
\hline Posterior & $1(1.69)$ & $3(3.09)$ & $4(2.56)$ & \\
\hline Corpus + posterior & $10(16.95)$ & $14(14.43)$ & $24(15.38)$ & \\
\hline
\end{tabular}

${ }^{\mathrm{a}}$ Data are given as mean \pm standard deviation for continuous variables and as frequency (percentage) for categorical variables. 


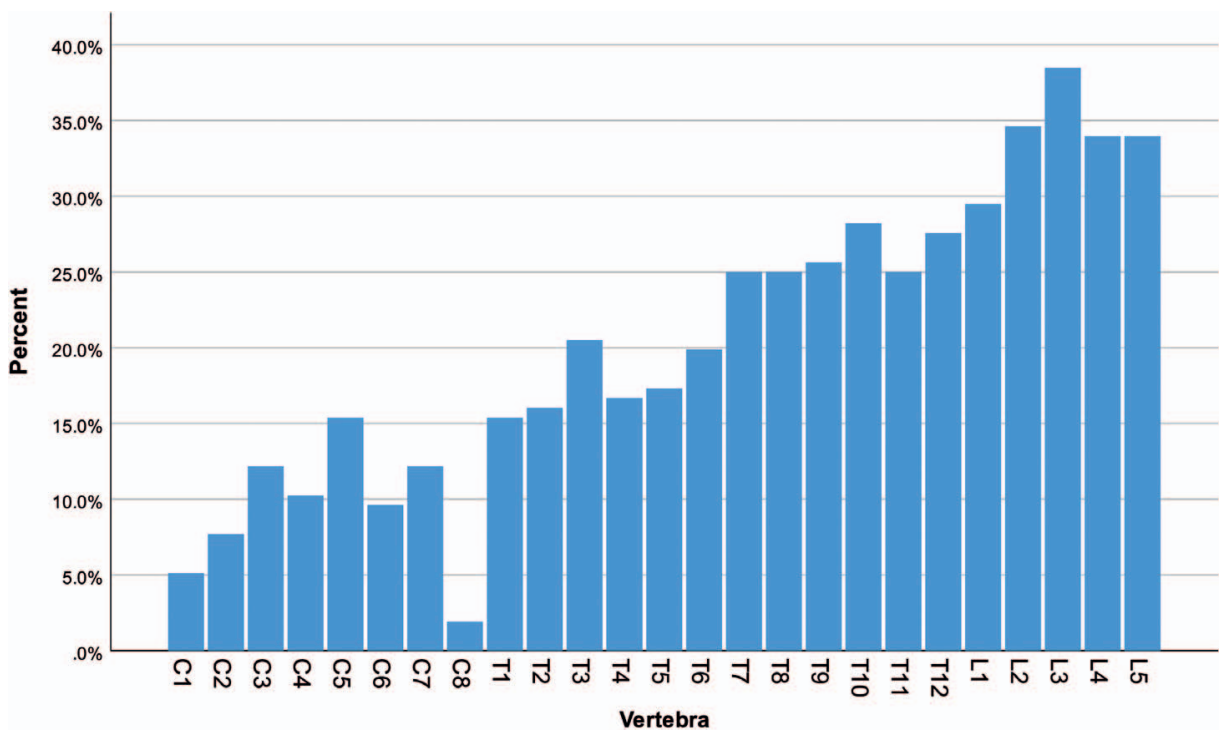

Figure. Percentages of each spinal metastasis.

were on corpus, $4(2.56 \%)$ on posterior, and 10 $(6.41 \%)$ on both. Metastasis was most common on C5 $(23.73 \%)$ in females, while most common on C7 $(15.46 \%)$ in males. C5 spinal metastasis percentage was significantly higher in females than in males $(P$ $=.043$; Table 1). No significant differences existed between genders regarding presence of other cervical spinal metastases (Table 1).

One hundred and twenty $(76.92 \%)$ patients had thoracic spinal metastases. Metastasis was most common on $\mathrm{T} 10(28.21 \%)$ among all patients. Twenty-six $(16.67 \%)$ of them were on corpus, 4 $(2.56 \%)$ on posterior, and $14(8.97 \%)$ on both. Metastasis was most common on T8 $(35.59 \%)$ in females, while most common on T9 $(26.80 \%)$ in males. T8 spinal metastasis percentage was significantly higher in females than in males $(P=.028$; Table 1). No significant differences existed between genders regarding presence of other thoracic spinal metastases (Table 1).

One hundred and nine $(69.87 \%)$ patients had lumbar spinal metastases. Metastasis was most common on L3 (38.46\%) among all patients. Twenty-nine $(18.59 \%)$ of them were on corpus, 8 $(5.13 \%)$ on posterior, and $23(14.74 \%)$ on both. Metastasis was most common on L3 both in females $(45.76 \%)$ and in males $(34.02 \%)$. L1 spinal metastasis percentage was significantly higher in females $(42.37 \%)$ than in males $(21.65 \% ; P=.010$; Table 1$)$. No significant differences existed between genders regarding presence of other lumbar spinal metastases (Table 1). Each spinal metastasis is shown in the Figure.

Patients with prostate cancer were significantly older than patients with respiratory system, breast, gastrointestinal tract, and gynecologic cancers $(P=$

Table 2. Summary of age and metastasis characteristics regarding cancer types., ${ }^{a, b}$

\begin{tabular}{|c|c|c|c|c|c|c|c|}
\hline & $\begin{array}{c}\text { Respiratory } \\
\text { System } \\
(n=74)\end{array}$ & $\begin{array}{c}\text { Breast } \\
(n=33)\end{array}$ & $\begin{array}{c}\text { Gastrointestinal } \\
\text { Tract } \\
(n=16)\end{array}$ & $\begin{array}{c}\text { Urinary } \\
\text { Tract } \\
(n=10)\end{array}$ & $\begin{array}{l}\text { Prostate } \\
(n=18)\end{array}$ & $\begin{array}{l}\text { Gynecologic } \\
\quad(n=5)\end{array}$ & $\boldsymbol{P}$ \\
\hline Age & $63.82 \pm 11.26 \mathrm{~A}$ & $62.82 \pm 14.27 \mathrm{~A}$ & $61.88 \pm 11.32 \mathrm{~A}$ & $72.10 \pm 12.11 \mathrm{AB}$ & $74.67 \pm 8.95 \mathrm{~B}$ & $64.40 \pm 4.22 \mathrm{~A}$ & .001 \\
\hline \multicolumn{8}{|l|}{ Metastasis status } \\
\hline Single & $20(27.03)$ & $4(12.12)$ & $8(50.00)$ & $4(40.00)$ & $3(16.67)$ & $1(20.00)$ & .071 \\
\hline Multiple & $54(72.97)$ & $29(87.88)$ & $8(50.00)$ & $6(60.00)$ & $15(83.33)$ & $4(80.00)$ & \\
\hline \multicolumn{8}{|l|}{ Region } \\
\hline Cervical & $1(1.35)$ & $1(3.03)$ & $1(6.25)$ & $1(10.00)$ & $0(0.00)$ & $0(0.00)$ & .313 \\
\hline Thoracic & $21(28.38)$ & $3(9.09)$ & $2(12.50)$ & $2(20.00)$ & $2(11.11)$ & $1(20.00)$ & \\
\hline Lumbar & $10(13.51)$ & $3(9.09)$ & $6(37.50)$ & $3(30.00)$ & $4(22.22)$ & $1(20.00)$ & \\
\hline Cervical + thoracic & $7(9.46)$ & $2(6.06)$ & $1(6.25)$ & $2(20.00)$ & $0(0.00)$ & $0(0.00)$ & \\
\hline Cervical + lumbar & $3(4.05)$ & $2(6.06)$ & $0(0.00)$ & $0(0.00)$ & $0(0.00)$ & $0(0.00)$ & \\
\hline Thoracic + lumbar & $17(22.97)$ & $14(42.42)$ & $2(12.50)$ & $1(10.00)$ & $9(50.00)$ & $2(40.00)$ & \\
\hline Cervical + thoracic + lumbar & $15(20.27)$ & $8(24.24)$ & $4(25.00)$ & $1(10.00)$ & $3(16.67)$ & $1(20.00)$ & \\
\hline
\end{tabular}

${ }^{a}$ Data are given as mean \pm standard deviation for continuous variables and as frequency (percentage) for categorical variables.

${ }^{\mathrm{b}} \mathrm{S}$ ame letters denote the lack of a statistically significant difference between groups. 
Table 3. Summary of cervical spinal metastasis with regard to cancer types. ${ }^{\text {a,b }}$

\begin{tabular}{|c|c|c|c|c|c|c|c|}
\hline & $\begin{array}{c}\text { Respiratory } \\
\text { System } \\
(\mathrm{n}=74)\end{array}$ & $\begin{array}{c}\text { Breast } \\
(n=33)\end{array}$ & $\begin{array}{c}\text { Gastrointestinal } \\
\text { Tract } \\
(\mathbf{n}=16)\end{array}$ & $\begin{array}{c}\text { Urinary } \\
\text { Tract } \\
(n=10)\end{array}$ & $\begin{array}{l}\text { Prostate } \\
(\mathrm{n}=18)\end{array}$ & $\begin{array}{l}\text { Gynecologic } \\
(n=5)\end{array}$ & $P$ \\
\hline Cervical spine & $26(35.14)$ & $13(39.39)$ & $6(37.50)$ & $4(40.00)$ & $3(16.67)$ & $1(20.00)$ & .613 \\
\hline $\mathrm{C} 1$ & $4(5.41)$ & $3(9.09)$ & $0(0.00)$ & $0(0.00)$ & $0(0.00)$ & $1(20.00)$ & .334 \\
\hline Corpus & $2(2.70)$ & $1(3.03)$ & $0(0.00)$ & $0(0.00)$ & $0(0.00)$ & $1(20.00)$ & \\
\hline Posterior & $0(0.00)$ & $1(3.03)$ & $0(0.00)$ & $0(0.00)$ & $0(0.00)$ & $0(0.00)$ & \\
\hline Corpus + posterior & $2(2.70)$ & $1(3.03)$ & $0(0.00)$ & $0(0.00)$ & $0(0.00)$ & $0(0.00)$ & \\
\hline $\mathrm{C} 2$ & $7(9.46)$ & $2(6.06)$ & $1(6.25)$ & $1(10.00)$ & $0(0.00)$ & $1(20.00)$ & .679 \\
\hline Corpus & $4(5.41)$ & $1(3.03)$ & $0(0.00)$ & $1(10.00)$ & $0(0.00)$ & $1(20.00)$ & \\
\hline Posterior & $0(0.00)$ & $0(0.00)$ & $0(0.00)$ & $0(0.00)$ & $0(0.00)$ & $0(0.00)$ & \\
\hline Corpus + posterior & $3(4.05)$ & $1(3.03)$ & $1(6.25)$ & $0(0.00)$ & $0(0.00)$ & $0(0.00)$ & \\
\hline C3 & $6(8.11)$ & $9(27.27)$ & $1(6.25)$ & $1(10.00)$ & $2(11.11)$ & $0(0.00)$ & .092 \\
\hline Corpus & $2(2.70)$ & $3(9.09)$ & $0(0.00)$ & $1(10.00)$ & $0(0.00)$ & $0(0.00)$ & \\
\hline Posterior & $1(1.35)$ & $1(3.03)$ & $0(0.00)$ & $0(0.00)$ & $0(0.00)$ & $0(0.00)$ & \\
\hline Corpus + posterior & $3(4.05)$ & $5(15.15)$ & $1(6.25)$ & $0(0.00)$ & $2(11.11)$ & $0(0.00)$ & \\
\hline $\mathrm{C} 4$ & $6(8.11)$ & $6(18.18)$ & $1(6.25)$ & $0(0.00)$ & $3(16.67)$ & $0(0.00)$ & .367 \\
\hline Corpus & $4(5.41)$ & $1(3.03)$ & $0(0.00)$ & $0(0.00)$ & $2(11.11)$ & $0(0.00)$ & \\
\hline Posterior & $1(1.35)$ & $1(3.03)$ & $0(0.00)$ & $0(0.00)$ & $0(0.00)$ & $0(0.00)$ & \\
\hline Corpus + posterior & $1(1.35)$ & $4(12.12)$ & $1(6.25)$ & $0(0.00)$ & $1(5.56)$ & $0(0.00)$ & \\
\hline $\mathrm{C} 5$ & $5(6.76) \mathrm{A}$ & $10(30.30) \mathrm{B}$ & $4(25.00) \mathrm{B}$ & $2(20.00) \mathrm{AB}$ & $2(11.11) \mathrm{AB}$ & $1(20.00) \mathrm{AB}$ & .042 \\
\hline Corpus & $3(4.05)$ & $2(6.06)$ & $1(6.25)$ & $1(10.00)$ & $2(11.11)$ & $1(20.00)$ & \\
\hline Posterior & $0(0.00)$ & $3(9.09)$ & $1(6.25)$ & $0(0.00)$ & $0(0.00)$ & $0(0.00)$ & \\
\hline Corpus + posterior & $2(2.70)$ & $5(15.15)$ & $2(12.5)$ & $1(10.00)$ & $0(0.00)$ & $0(0.00)$ & \\
\hline C6 & $7(9.46)$ & $4(12.12)$ & $2(12.5)$ & $1(10.00)$ & $1(5.56)$ & $0(0.00)$ & .938 \\
\hline Corpus & $3(4.05)$ & $1(3.03)$ & $2(12.5)$ & $1(10.00)$ & $1(5.56)$ & $0(0.00)$ & \\
\hline Posterior & $2(2.70)$ & $1(3.03)$ & $0(0.00)$ & $0(0.00)$ & $0(0.00)$ & $0(0.00)$ & \\
\hline Corpus + posterior & $2(2.70)$ & $2(6.06)$ & $0(0.00)$ & $0(0.00)$ & $0(0.00)$ & $0(0.00)$ & \\
\hline $\mathrm{C} 7$ & $11(14.86)$ & $3(9.09)$ & $3(18.75)$ & $0(0.00)$ & $1(5.56)$ & $1(20.00)$ & .571 \\
\hline Corpus & $4(5.41)$ & $1(3.03)$ & $1(6.25)$ & $0(0.00)$ & $1(5.56)$ & $0(0.00)$ & \\
\hline Posterior & $4(5.41)$ & $0(0.00)$ & $2(12.50)$ & $0(0.00)$ & $0(0.00)$ & $0(0.00)$ & \\
\hline Corpus + posterior & $3(4.05)$ & $2(6.06)$ & $0(0.00)$ & $0(0.00)$ & $0(0.00)$ & $1(20.00)$ & \\
\hline $\mathrm{C} 8$ & $1(1.35)$ & $1(3.03)$ & $0(0.00)$ & $0(0.00)$ & $1(5.56)$ & $0(0.00)$ & .819 \\
\hline Corpus & $1(1.35)$ & $0(0.00)$ & $0(0.00)$ & $0(0.00)$ & $1(5.56)$ & $0(0.00)$ & \\
\hline Posterior & $0(0.00)$ & $0(0.00)$ & $0(0.00)$ & $0(0.00)$ & $0(0.00)$ & $0(0.00)$ & \\
\hline Corpus + posterior & $0(0.00)$ & $1(3.03)$ & $0(0.00)$ & $0(0.00)$ & $0(0.00)$ & $0(0.00)$ & \\
\hline
\end{tabular}

${ }^{\text {a}}$ Data are given as mean \pm standard deviation for continuous variables and as frequency (percentage) for categorical variables.

${ }^{\mathrm{b}} \mathrm{Same}$ letters denote the lack of a statistically significant difference between groups.

.001; Table 2). No significant differences existed between other cancer types regarding age. Fifty-four $(72.97 \%)$ patients with respiratory system cancers had multiple metastases, while $29(87.88 \%)$ patients with breast cancer, $8(50.00 \%)$ patients with gastrointestinal tract cancers, $6(60.00 \%)$ patients with urinary tract cancers, $15(83.33 \%)$ patients with prostate cancer, and $4(80.00 \%)$ patients with gynecologic cancers had multiple metastases $(P=$ .071; Table 2). The most common metastasis region was the thoracic spine in the respiratory system cancers $(28.38 \%)$, the thoracic + lumbar spine in breast $(42.42 \%)$, prostate $(50.00 \%)$, and gynecologic $(40.00 \%)$ cancers, and the lumbar spine in gastrointestinal $(37.50 \%)$ and urinary $(30.00 \%)$ tract cancers $(P=.313$; Table 2$)$.

Twenty-six $(35.14 \%)$ patients with respiratory system cancers had metastasis on the cervical spine, while $13(39.39 \%)$ patients with breast cancer, 6 $(37.50 \%)$ patients with gastrointestinal tract cancers, $4(40.00 \%)$ patients with urinary tract cancers, $3(16.67 \%)$ patients with prostate cancer, and 1
$(20.00 \%)$ patient with gynecologic cancer had metastases on the cervical spine $(P=.613$; Table $3)$. Respiratory system cancer metastasis was most common on C7 $(14.86 \%)$. Four $(5.41 \%)$ of them were on corpus, $4(5.41 \%)$ on posterior, and 3 $(4.05 \%)$ on both. Breast cancer metastasis was most common on C5 $(30.30 \%)$. Two $(6.06 \%)$ of them were on corpus, $3(9.09 \%)$ on posterior, and 5 $(15.15 \%)$ on both. Gastrointestinal tract cancer metastasis was most common on C5 $(25.00 \%)$. One $(6.25 \%)$ of them was on corpus, $1(6.25 \%)$ on posterior, and $2(12.5 \%)$ on both. Urinary tract cancer metastasis was most common on C5 $(20.00 \%)$. One $(10.00 \%)$ of them was on corpus and $1(10.00 \%)$ on both corpus and posterior. Prostate cancer metastasis was most common on C4 $(16.67 \%)$. Two $(11.11 \%)$ of them were on corpus and $1(5.56 \%)$ on both corpus and posterior. C5 spinal metastasis percentages were significantly higher in breast and gastrointestinal tract cancers than in respiratory system cancers $(P=.042$; Table $3)$. No significant differences existed between cancer 
Table 4. Summary of thoracic spinal metastasis with regard to cancer types. ${ }^{a}$

\begin{tabular}{|c|c|c|c|c|c|c|c|}
\hline & $\begin{array}{c}\text { Respiratory } \\
\text { System } \\
(n=74)\end{array}$ & $\begin{array}{c}\text { Breast } \\
(n=33)\end{array}$ & $\begin{array}{c}\text { Gastrointestinal } \\
\text { Tract } \\
(\mathbf{n}=16)\end{array}$ & $\begin{array}{c}\text { Urinary } \\
\text { Tract } \\
(n=10)\end{array}$ & $\begin{array}{l}\text { Prostate } \\
(\mathrm{n}=18)\end{array}$ & $\begin{array}{l}\text { Gynecologic } \\
(n=5)\end{array}$ & $P$ \\
\hline Thoracic spine & $60(81.08)$ & $27(81.82)$ & $9(56.25)$ & $6(60.00)$ & $14(77.78)$ & $4(80.00)$ & .247 \\
\hline $\mathrm{T} 1$ & $13(17.57)$ & $4(12.12)$ & $3(18.75)$ & $0(0.00)$ & $3(16.67)$ & $1(20.00)$ & .761 \\
\hline Corpus & $9(12.16)$ & $1(3.03)$ & $2(12.50)$ & $0(0.00)$ & $2(11.11)$ & $1(20.00)$ & \\
\hline Posterior & $3(4.05)$ & $0(0.00)$ & $0(0.00)$ & $0(0.00)$ & $0(0.00)$ & $0(0.00)$ & \\
\hline Corpus + posterior & $1(1.35)$ & $3(9.09)$ & $1(6.25)$ & $0(0.00)$ & $1(5.56)$ & $0(0.00)$ & \\
\hline $\mathrm{T} 2$ & $13(17.57)$ & $5(15.15)$ & $2(12.50)$ & $1(10.00)$ & $2(11.11)$ & $2(40.00)$ & .696 \\
\hline Corpus & $10(13.51)$ & $1(3.03)$ & $2(12.50)$ & $0(0.00)$ & $1(5.56)$ & $1(20.00)$ & \\
\hline Posterior & $1(1.35)$ & $1(3.03)$ & $0(0.00)$ & $1(10.00)$ & $0(0.00)$ & $1(20.00)$ & \\
\hline Corpus + posterior & $2(2.70)$ & $3(9.09)$ & $0(0.00)$ & $0(0.00)$ & $1(5.56)$ & $0(0.00)$ & \\
\hline $\mathrm{T} 3$ & $18(24.32)$ & $5(15.15)$ & $5(31.25)$ & $1(10.00)$ & $2(11.11)$ & $1(20.00)$ & .546 \\
\hline Corpus & $9(12.16)$ & $2(6.06)$ & $4(25.00)$ & $1(10.00)$ & $1(5.56)$ & $1(20.00)$ & \\
\hline Posterior & $1(1.35)$ & $0(0.00)$ & $0(0.00)$ & $0(0.00)$ & $0(0.00)$ & $0(0.00)$ & \\
\hline Corpus + posterior & $8(10.81)$ & $3(9.09)$ & $1(6.25)$ & $0(0.00)$ & $1(5.56)$ & $0(0.00)$ & \\
\hline $\mathrm{T} 4$ & $11(14.86)$ & $6(18.18)$ & $4(25.00)$ & $0(0.00)$ & $3(16.67)$ & $2(40.00)$ & .417 \\
\hline Corpus & $6(8.11)$ & $3(9.09)$ & $4(25.00)$ & $0(0.00)$ & $3(16.67)$ & $1(20.00)$ & \\
\hline Posterior & $2(2.70)$ & $1(3.03)$ & $0(0.00)$ & $0(0.00)$ & $0(0.00)$ & $1(20.00)$ & \\
\hline Corpus + posterior & $3(4.05)$ & $2(6.06)$ & $0(0.00)$ & $0(0.00)$ & $0(0.00)$ & $0(0.00)$ & \\
\hline $\mathrm{T} 5$ & $8(10.81) \mathrm{A}$ & $7(21.21) \mathrm{A}$ & $4(25.00) \mathrm{A}$ & $0(0.00) \mathrm{A}$ & $4(22.22) \mathrm{A}$ & $4(80.00) \mathrm{B}$ & .002 \\
\hline Corpus & $2(2.70)$ & $3(9.09)$ & $4(25.00)$ & $0(0.00)$ & $3(16.67)$ & $3(60.00)$ & \\
\hline Posterior & $0(0.00)$ & $1(3.03)$ & $0(0.00)$ & $0(0.00)$ & $1(5.56)$ & $1(20.00)$ & \\
\hline Corpus + posterior & $6(8.11)$ & $3(9.09)$ & $0(0.00)$ & $0(0.00)$ & $0(0.00)$ & $0(0.00)$ & \\
\hline T6 & $9(12.16)$ & $10(30.30)$ & $3(18.75)$ & $2(20.00)$ & $6(33.33)$ & $1(20.00)$ & .215 \\
\hline Corpus & $7(9.46)$ & $7(21.21)$ & $2(12.50)$ & $2(20.00)$ & $2(11.11)$ & $1(20.00)$ & \\
\hline Posterior & $0(0.00)$ & $1(3.03)$ & $0(0.00)$ & $0(0.00)$ & $0(0.00)$ & $0(0.00)$ & \\
\hline Corpus + posterior & $2(2.70)$ & $2(6.06)$ & $1(6.25)$ & $0(0.00)$ & $4(22.22)$ & $0(0.00)$ & \\
\hline $\mathrm{T} 7$ & $17(22.97)$ & $9(27.27)$ & $3(18.75)$ & $3(30.00)$ & $6(33.33)$ & $1(20.00)$ & .918 \\
\hline Corpus & $14(18.92)$ & $7(21.21)$ & $2(12.50)$ & $2(20.00)$ & $3(16.67)$ & $1(20.00)$ & \\
\hline Posterior & $0(0.00)$ & $0(0.00)$ & $0(0.00)$ & $1(10.00)$ & $0(0.00)$ & $0(0.00)$ & \\
\hline Corpus + posterior & $3(4.05)$ & $2(6.06)$ & $1(6.25)$ & $0(0.00)$ & $3(16.67)$ & $0(0.00)$ & \\
\hline $\mathrm{T} 8$ & $15(20.27)$ & $13(39.39)$ & $2(12.50)$ & $1(10.00)$ & $7(38.89)$ & $1(20.00)$ & .110 \\
\hline Corpus & $9(12.16)$ & $9(27.27)$ & $2(12.50)$ & $0(0.00)$ & $4(22.22)$ & $1(20.00)$ & \\
\hline Posterior & $1(1.35)$ & $1(3.03)$ & $0(0.00)$ & $0(0.00)$ & $0(0.00)$ & $0(0.00)$ & \\
\hline Corpus + posterior & $5(6.76)$ & $3(9.09)$ & $0(0.00)$ & $1(10.00)$ & $3(16.67)$ & $0(0.00)$ & \\
\hline T9 & $20(27.03)$ & $9(27.27)$ & $1(6.25)$ & $1(10.00)$ & $8(44.44)$ & $1(20.00)$ & .157 \\
\hline Corpus & $15(20.27)$ & $8(24.24)$ & $1(6.25)$ & $1(10.00)$ & $3(16.67)$ & $1(20.00)$ & \\
\hline Posterior & $2(2.70)$ & $0(0.00)$ & $0(0.00)$ & $0(0.00)$ & $1(5.56)$ & $0(0.00)$ & \\
\hline Corpus + posterior & $3(4.05)$ & $1(3.03)$ & $0(0.00)$ & $0(0.00)$ & $4(22.22)$ & $0(0.00)$ & \\
\hline $\mathrm{T} 10$ & $16(21.62) \mathrm{A}$ & $13(39.39) \mathrm{AB}$ & $2(12.50) \mathrm{A}$ & $1(10.00) \mathrm{A}$ & $9(50.00) \mathrm{B}$ & $3(60.00) \mathrm{B}$ & .016 \\
\hline Corpus & $9(12.16)$ & $9(27.27)$ & $1(6.25)$ & $0(0.00)$ & $4(22.22)$ & $3(60.00)$ & \\
\hline Posterior & $3(4.05)$ & $0(0.00)$ & $0(0.00)$ & $0(0.00)$ & $1(5.56)$ & $0(0.00)$ & \\
\hline Corpus + posterior & $4(5.41)$ & $4(12.12)$ & $1(6.25)$ & $1(10.00)$ & $4(22.22)$ & $0(0.00)$ & \\
\hline $\mathrm{T} 11$ & $17(22.97)$ & $5(15.15)$ & $4(25.00)$ & $2(20.00)$ & $8(44.44)$ & $3(60.00)$ & .113 \\
\hline Corpus & $11(14.86)$ & $1(3.03)$ & $2(12.50)$ & $1(10.00)$ & $4(22.22)$ & $3(60.00)$ & \\
\hline Posterior & $3(4.05)$ & $0(0.00)$ & $0(0.00)$ & $0(0.00)$ & $0(0.00)$ & $0(0.00)$ & \\
\hline Corpus + posterior & $3(4.05)$ & $4(12.12)$ & $2(12.5)$ & $1(10.00)$ & $4(22.22)$ & $0(0.00)$ & \\
\hline $\mathrm{T} 12$ & 17 (22.97) & $12(36.36)$ & $4(25.00)$ & $2(20.00)$ & $7(38.89)$ & $1(20.00)$ & .593 \\
\hline Corpus & $9(12.16)$ & $3(9.09)$ & $1(6.25)$ & $0(0.00)$ & $2(11.11)$ & $1(20.00)$ & \\
\hline Posterior & $1(1.35)$ & $1(3.03)$ & $0(0.00)$ & $1(10.00)$ & $0(0.00)$ & $0(0.00)$ & \\
\hline Corpus + posterior & $7(9.46)$ & $8(24.24)$ & $3(18.75)$ & $1(10.00)$ & $5(27.78)$ & $0(0.00)$ & \\
\hline
\end{tabular}

${ }^{a}$ Data are given as mean \pm standard deviation for continuous variables and as frequency (percentage) for categorical variables.

${ }^{\mathrm{b}} \mathrm{S}$ ame letters denote the lack of a statistically significant difference between groups.

types regarding presence of other cervical spinal metastases (Table 3).

Sixty $(81.08 \%)$ patients with respiratory system cancers had metastases on the thoracic spine, while $27(81.82 \%)$ patients with breast cancers, 9 $(56.25 \%)$ patients with gastrointestinal tract cancers, $6(60.00 \%)$ patients with urinary tract cancers, $14(77.78 \%)$ patients with prostate cancer, and 4 $(80.00 \%)$ patients with gynecologic cancers had metastases on the thoracic spine $(P=.247$; Table 4$)$. Respiratory system cancer metastasis was most common on T9 $(27.03 \%)$. Fifteen $(20.27 \%)$ of them were on corpus, $2(2.70 \%)$ on posterior, and 3 $(4.05 \%)$ on both. Breast cancer metastasis was most common on T8 $(39.39 \%)$ and T10 $(39.39 \%)$. Nine $(27.27 \%)$ of the T8s were on corpus, $1(3.03 \%)$ on posterior, and $3(9.09 \%)$ on both, while $9(27.27 \%)$ of T10s were on corpus and $4(12.12 \%)$ on both corpus and posterior. Gastrointestinal tract cancer metastasis was most common on T3 $(31.25 \%)$. Four $(25.00 \%)$ of them were on corpus and $1(6.25 \%)$ on both corpus and posterior. Urinary tract cancer 
Table 5. Summary of lumbar spinal metastasis with regard to cancer types. ${ }^{a}$

\begin{tabular}{|c|c|c|c|c|c|c|c|}
\hline & $\begin{array}{c}\text { Respiratory } \\
\text { System } \\
(\mathrm{n}=74)\end{array}$ & $\begin{array}{c}\text { Breast } \\
(\mathrm{n}=\mathbf{3 3})\end{array}$ & $\begin{array}{c}\text { Gastrointestinal } \\
\text { Tract } \\
(\mathrm{n}=16)\end{array}$ & $\begin{array}{c}\text { Urinary } \\
\text { Tract } \\
(\mathrm{n}=10)\end{array}$ & $\begin{array}{l}\text { Prostate } \\
(\mathrm{n}=18)\end{array}$ & $\begin{array}{c}\text { Gynecologic } \\
(\mathrm{n}=\mathbf{5})\end{array}$ & $P$ \\
\hline Lumbar spine & $45(60.81)$ & $27(81.82)$ & $12(75.00)$ & $5(50.00)$ & $16(88.89)$ & $4(80.00)$ & .061 \\
\hline L1 & $15(20.27) \mathrm{A}$ & $18(54.55) \mathrm{B}$ & $3(18.75) \mathrm{A}$ & $2(20.00) \mathrm{A}$ & 7 (38.89)AB & $1(20.00) \mathrm{A}$ & .009 \\
\hline Corpus & $9(12.16)$ & $9(27.27)$ & $0(0.00)$ & $1(10.00)$ & $2(11.11)$ & $0(0.00)$ & \\
\hline Posterior & $2(2.70)$ & $4(12.12)$ & $0(0.00)$ & $1(10.00)$ & $0(0.00)$ & $0(0.00)$ & \\
\hline Corpus + posterior & $4(5.41)$ & $5(15.15)$ & $3(18.75)$ & $0(0.00)$ & $5(27.78)$ & $1(20.00)$ & \\
\hline L2 & $21(28.38) \mathrm{A}$ & $16(48.48) \mathrm{B}$ & $4(25.00) \mathrm{A}$ & $0(0.00) \mathrm{A}$ & $10(55.56) \mathrm{B}$ & $3(60.00) \mathrm{B}$ & .011 \\
\hline Corpus & $11(14.86)$ & $8(24.24)$ & $1(6.25)$ & $0(0.00)$ & $2(11.11)$ & $1(20.00)$ & \\
\hline Posterior & $3(4.05)$ & $3(9.09)$ & $0(0.00)$ & $0(0.00)$ & $1(5.56)$ & $0(0.00)$ & \\
\hline Corpus + posterior & $7(9.46)$ & $5(15.15)$ & $3(18.75)$ & $0(0.00)$ & $7(38.89)$ & $2(40.00)$ & \\
\hline L3 & $25(33.78)$ & $18(54.55)$ & $4(25.00)$ & $3(30.00)$ & $9(50.00)$ & $1(20.00)$ & .183 \\
\hline Corpus & $16(21.62)$ & $7(21.21)$ & $2(12.50)$ & $2(20.00)$ & $2(11.11)$ & $0(0.00)$ & \\
\hline Posterior & $3(4.05)$ & $3(9.09)$ & $0(0.00)$ & $1(10.00)$ & $1(5.56)$ & $0(0.00)$ & \\
\hline Corpus + posterior & $6(8.11)$ & $8(24.24)$ & $2(12.50)$ & $0(0.00)$ & $6(33.33)$ & $1(20.00)$ & \\
\hline L4 & $19(25.68) \mathrm{A}$ & $16(48.48) \mathrm{B}$ & $7(43.75) \mathrm{AB}$ & $2(20.00) \mathrm{A}$ & $9(50.00) \mathrm{B}$ & $0(0.00) \mathrm{A}$ & .041 \\
\hline Corpus & $9(12.16)$ & $6(18.18)$ & $4(25.00)$ & $1(10.00)$ & $4(22.22)$ & $0(0.00)$ & \\
\hline Posterior & $3(4.05)$ & $0(0.00)$ & $0(0.00)$ & $0(0.00)$ & $1(5.56)$ & $0(0.00)$ & \\
\hline Corpus + posterior & $7(9.46)$ & $10(30.30)$ & $3(18.75)$ & $1(10.00)$ & $4(22.22)$ & $0(0.00)$ & \\
\hline L5 & $22(29.73) \mathrm{A}$ & $12(36.36) \mathrm{A}$ & $4(25.00) \mathrm{A}$ & $1(10.00) \mathrm{A}$ & $12(66.67) \mathrm{B}$ & $2(40.00) \mathrm{AB}$ & .029 \\
\hline Corpus & $11(14.86)$ & $6(18.18)$ & $0(0.00)$ & $0(0.00)$ & $6(33.33)$ & $2(40.00)$ & \\
\hline Posterior & $3(4.05)$ & $0(0.00)$ & $0(0.00)$ & $0(0.00)$ & $1(5.56)$ & $0(0.00)$ & \\
\hline Corpus + posterior & $8(10.81)$ & $6(18.18)$ & $4(25.00)$ & $1(10.00)$ & $5(27.78)$ & $0(0.00)$ & \\
\hline
\end{tabular}

${ }^{a}$ Data are given as mean \pm standard deviation for continuous variables and as frequency (percentage) for categorical variables.

${ }^{\mathrm{b}} \mathrm{Same}$ letters denote the lack of a statistically significant difference between groups.

metastasis was most common on T7 (30.00\%). Two $(20.00 \%)$ of them were on corpus and $1(10.00 \%)$ on posterior. Prostate cancer metastasis was most common on T10 (50.00\%). Four $(22.22 \%)$ of them were on corpus, $1(5.56 \%)$ on posterior, and 4 $(22.22 \%)$ on both. Gynecologic cancer metastasis was most common on T5 $(80.00 \%)$. Three $(60.00 \%)$ of them were on corpus and $1(20.00 \%)$ on posterior. T5 spinal metastasis percentage was significantly higher in the gynecologic cancers than in the other cancers $(P=.002)$, while T10 spinal metastasis percentages were significantly higher in the prostate and gynecologic cancers than in the respiratory system, gastrointestinal, and urinary tract cancers $(P=.016)$. No significant differences existed between cancer types regarding presence of other thoracic spinal metastases (Table 4).

Forty-five $(60.81 \%)$ patients with respiratory system cancers had metastases on the lumbar spine, while $27(81.82 \%)$ patients with breast cancer, 12 $(75.00 \%)$ patients with gastrointestinal tract cancers, $5(50.00 \%)$ patients with urinary tract cancers, $16(88.89 \%)$ patients with prostate cancer, and 4 $(80.00 \%)$ patients with gynecologic cancers had metastases on the lumbar spine $(P=.061$; Table 5$)$. Respiratory system cancer metastasis was most common on L3 (33.78\%). Sixteen $(21.62 \%)$ of them were on corpus, $3(4.05 \%)$ on posterior, and 6 $(8.11 \%)$ on both. Breast cancer metastasis was most common on L1 $(54.55 \%)$ and L3 $(54.55 \%)$. Nine
(27.27\%) of L1s were on corpus, $4(12.12 \%)$ on posterior, and $5(15.15 \%)$ on both, while $7(21.21 \%)$ of L3s were on corpus, $3(9.09 \%)$ on posterior, and $8(24.24 \%)$ on both. Gastrointestinal tract cancer metastasis was most common on L4 $(43.75 \%)$. Four $(25.00 \%)$ of them were on corpus and $3(18.75 \%)$ on both corpus and posterior. Urinary tract cancer metastasis was most common on L3 $(30.00 \%)$. Two $(20.00 \%)$ of them were on corpus and $1(10.00 \%)$ on posterior. Prostate cancer metastasis was most common on L5 (66.67\%). Six $(33.33 \%)$ of them were on corpus, $1(5.56 \%)$ on posterior, and 5 $(27.78 \%)$ on both. Gynecologic cancer metastasis was most common on L2 $(60.00 \%)$. One $(20.00 \%)$ of them was on corpus and $2(40.00 \%)$ on both corpus and posterior. L1 spinal metastasis percentage was significantly higher in breast cancer than in respiratory system, gastrointestinal tract, urinary tract, and gynecologic cancers $(P=.009)$. L2 spinal metastasis percentages were significantly higher in breast, prostate, and gynecologic cancers than in respiratory system, gastrointestinal tract, and urinary tract cancers $(P=.011)$. L4 spinal metastasis percentages were significantly higher in breast and prostate cancers than in respiratory system, urinary tract, and gynecological cancers $(P=.041)$. L5 spinal metastasis percentage was significantly higher in prostate cancer than in respiratory system, breast, gastrointestinal tract, and urinary tract cancers $(P=$ .029). No significant differences existed between 
cancer types regarding presence of L3 spinal metastases (Table 5).

\section{DISCUSSION}

In this study, clinical data of 156 patients with isolated spinal metastasis without visceral organ metastasis were analyzed retrospectively, including demographic characteristics (age, gender), pathological diagnostic reports, and PET-CT results. Spinal involvement characteristics of primary cancers were evaluated by analyzing both the main spine structures and specific locations in each. Although not statistically significant, respiratory system cancers metastasize to the thoracic spine $(23.38 \%)$, breast cancer metastasizes to the thoracic + lumbal spine $(42.42 \%)$, gastrointestinal $(37.5 \%)$ and urinary $(30 \%)$ tract cancers metastasize to the lumbal spine, and prostate $(50.0 \%)$ and gynecological $(40 \%)$ cancers metastasize to the thoracic + lumbal spine commonly. These statistics suggest that a primary cancer tends to metastasize to a given spine section and specific location within. The C5 vertebrawas the most frequently involved spine in the cervical spine. Respiratory system cancers most common ly metastasized to the corpus or posterior of the $\mathrm{C} 4$ spine, and prostate cancers metastasized to the corpus of the $\mathrm{C} 4$ spine. Breast and urinary tract cancers were statistically significantly more metastasized to the $\mathrm{C} 5$ spine than other cancers $(P$ $<.05$; Table 3). While breast cancer spread most commonly to the corpus + posterior of the C5 spine, urinary tract cancers have metastasized equally to the corpus and corpus + posterior regions of the C5 spine. As a result of the present study, while respiratory system cancers metastasized to the corpus of the T9 spine in the thoracic spine commonly, breast cancer metastasized to the corpus of the T8 and T10 spine, gastrointestinal tract cancers metastasized to the corpus of the T3 spine, urinary tract cancers metastasized to the corpus of the T3 spine, prostate cancer spread to the corpus and corpus + posterior of the T10 spine, and gynecological cancers metastasized to the corpus of the T5 spine most commonly. It has been observed that gynecological cancers statistically significantly metastasized to the T5 spine, and prostate cancer metastasized to the T10 spine more than other cancers $(P<.05$; Table 4$)$. In the lumbar spine, it has been observed that breast cancer statistically significantly metastasized to the L1 spine, breast, prostate, and gynecological cancers to the L2 spine, breast and prostate cancer to the L4 spine, and prostate cancer to the L5 spine more than other cancers $(P<.05$; Table 5$)$.

The type and location of the primary cancer is unknown in $3 \%-15 \%$ of all patients with metastatic carcinoma, and approximately $5 \%-20 \%$ of these patients constitute the first detectable lesion bone metastases. ${ }^{20-23}$ Spinal metastases have been reported to account for approximately $80 \%$ of cancers of unknown primary. ${ }^{20,24}$ Therefore, it is very important to determine the distribution properties of bone metastases. I believe that the data in this study will be beneficial in evaluating cancers of unknown primary that have metastasized to the spine.

Although authors have investigated spinal metastases in the literature, no authors have investigated isolated spinal metastases without visceral and additional bone metastases. Authors have shown that the thoracic spine is the most common metastasis localization as well as that the lumbar spine is the most common area of metastasis. ${ }^{25-27}$ The cervical spine is the area with the least metastasis. ${ }^{28}$ In the present study, the thoracic + lumbal spine was shown to be the most common area of metastasis. In accordance with the literature, the cervical spine is the place where metastasis was seen least. This study reveals the metastasis spread rates of each spine in terms of primary cancer, together with the main spinal sections, unlike existing studies in the literature.

Bone metastases of cancers can occur through systemic blood circulation or vertebral venous plexus. $^{7-9}$ Metastases that spread using the blood circulatory system can spread to any bone, especially to extremities, and this is considered to occur randomly. ${ }^{16-18}$ Diffusion from the venous system is the main process of spinal metastasis, and it rarely reaches the extremities by using the venous system. ${ }^{16-18}$ Therefore, patients with extremity, visceral, or metastases to both were excluded from the present study to examine spinal metastases that occurred specifically through the vertebral venous system. In this way, I thought the spinal metastasis characteristics of the vertebral venous system could be better understood. Few studies are available in which authors investigate metastases through the vertebral venous plexus. ${ }^{18,19}$ In the study of Zhu et al, ${ }^{18}$ bone metastases in the whole body without visceral metastasis were investigated, and they stated that the primary cancer tends to metastasize to the bone close to it. ${ }^{18}$ Although similar results are 
available in this study, the main difference between this study and the study of Zhu et $\mathrm{al}^{18}$ is the detailed evaluation of isolated spinal metastases. Wang et $\mathrm{al}^{19}$ investigated bone metastases of lung and prostate cancers in their study. ${ }^{19}$ Authors of publications in the literature have examined bone metastases for a specific type of cancer, such as the study of Wang et al. ${ }^{19,29-32}$ This study evaluates spinal metastasis features by vertebral venous plexus in detail by examining cancers in all body regions.

This study has some limitations. First, the most important limitation is that the majority of the patients in the study had respiratory system and breast cancers. It should be taken into consideration that both cancers are the most common cancers in the society in this case. However, studies with a higher number of patients are needed for more objective results.

\section{CONCLUSIONS}

Although the vertebral venous system is an important pathway for spinal metastasis formation, its characteristic features have not been revealed clearly yet. As a result of this study, primary cancers without visceral metastasis tend to metastasize to the near spine. Comprehensive studies involving more patients are needed to reveal the mechanism of metastasis formation with the vertebral venous system more clearly. The results obtained by detailed examination of spinal metastases may provide effective and useful clues in finding cancers of unknown primary.

\section{REFERENCES}

1. Mundy GR. Metastasis to bone: causes, consequences and therapeutic opportunities. Nat Rev Cancer. 2002;2(8):584-593. doi: $10.1038 / \operatorname{nrc} 867$

2. Macedo F, Ladeira K, Pinho F, et al. Bone metastasis; an overview. Oncol Rev. 2017;11(1):321. doi:10.4081/oncol.2017. 321

3. Coleman R. Metastatic bone disease: clinical features, pathophysiology and treatment strategies. Cancer Treat Rev. 2001;27(3):165-176. doi: 10.1053/ctrv.2000.0210

4. Shaw B, Mansfield FL, Borges L. One-stage posterolateral decompression and stabilization for primary and metastatic vertebral tumors in the thoracic and lumbar spine. J Neurosurg. 1989;7(3):405-410. doi:10.3171/jns.1989.70.3.0405

5. Abeloff MD, Armitage JO, Niederhuber JE, et al. Abeloff's Clinical Oncology. 4th ed. Philadelphia, PA: Churchill Livingstone Elsevier; 2008.

6. Coleman RE. Clinical features of metastatic bone disease and risk of skeletal morbidity. Clin Cancer Res. 2006;12(12):6243-6649. doi:10.1158/1078-0432.CCR-06-0931

7. Lewandrowski KU, Anderson ME, McLain RF. Tumors of the spine. In: Herkowitz HN, Garfin SR, Eismont FJ, Bell GR, Balderston RA, eds. Rothman-Simeone, The Spine. Philadelphia, PA: Elsevier Saunders; 2011:1480-1512.

8. Hatrick NC, Lucas JD, Timothy AR, et al. The surgical treatment of metastatic disease of the spine. Radiother Oncol. 2000;56(3):335-339. doi:10.1016/S0167-8140(00)00199-7

9. Choi D, Crockard A, Bunger C, et al. Review of metastatic spine tumour classification and indications for surgery: the consensus statement of the Global Spine Tumour Study Group. Eur Spine J. 2010;19(2):215-222. doi:10.1007/ s00586-009-1252-x

10. Lun D, Chen NW, Feng J, et al. Visceral metastasis: a prognostic factor of survival in patients with spinal metastases. Orthop Surg. 2020;12(2):552-560. doi:10.1111/os.12657

11. Byrne TN. Spinal cord compression from epidural metastases. N Engl J Med. 1992;327(9):614-619. doi:10.1056/ NEJM199208273270907

12. Aboulaflia AJ, Levine AM. Musculoskeletal and metastatic tumors. In: Fardon DF, Garfin SR, eds. Orthopaedic Knowledge Update: Spine 2. Rosemont, IL: American Academy of Orthopaedic Surgeons, 2002;411-431.

13. Cecchini M, Wetterwald A, Pluijm G, et al. Molecular and biological mechanisms of bone metastasis. EAU Update Series. 2005;3(4):214-226. doi:10.1016/j.euus.2005.09.006

14. Selvaggi G, Scagliotti G. Management of bone metastases in cancer: a review. Clin Rev Oncol Hematol. 2005;56(3):365-378. doi:10.1016/j.critrevonc.2005.03.011

15. Clohisy D, Mantyh P. Bone cancer pain. Cancer. 2003;97(3):866. doi:10.1002/cncr.11144

16. Maccauro G, Spinelli MS, Mauro S, et al. Physiopathology of spine metastasis, Int J Surg Oncol. 2011;2011:107969. doi:10.1155/2011/107969

17. Kakhki VRD, Anvari K, Sadeghi R, et al. Pattern and distribution of bone metastases in common malignant tumors. Nuc Med Rev Cent East Eur. 2013;16(2):66-69. doi:10.5603/ NMR.2013.0037

18. Zhu M, Liu X, Qu Y, et al. Bone metastasis pattern of cancer patients with bone metastasis but no visceral metastasis. J Bone Oncol. 2019;15:100219. doi:10.1016/j.jbo.2019.100219

19. Wang C, Shen Y, Zhu S. Distribution features of skeletal metastases: a comparative study between pulmonary and prostate cancers. Plos One. 2015;10(11):e0143437. doi:10.1371/ journal.pone.0143437

20. Barón MG, de la Gándara I, Espinosa E, et al. Bone metastases as the first manifestation of a tumour. Int Orthop. 1991;15(4):373-376. doi:10.1007/BF00186882

21. Jacobsen S, Stephensen SL, Paaske BP, et al. Skeletal metastases of unknown origin: a retrospective analysis of 29 cases. Acta Orthop Belg. 1997;63(1):15-22.

22. Katagiri H, Takahashi M, Inagaki J, et al. Determining the site of the primary cancer in patients with skeletal metastasis of unknown origin: a retrospective study. Cancer. 1999;86(3):533-537.

23. Destombe C, Botton E, Le Gal G, et al. Investigations for bone metastasis from an unknown primary. Joint Bone Spine. 2007;74(1):85-89. doi:10.1016/j.jbspin.2006.05.009

24. Nottebaert M, Exner GU, von Hochstetter AR, et al. Metastatic bone disease from occult carcinoma: a profile. Int Orthop. 1989;13(2):119-123. doi:10.1007/BF00266372

25. Jaffe WF. Tumors and Timorous Conditions of the Bones and Joints. Philadelphia PA: Lea and Febiger; 1958. 
26. Batson OV. The role of the vertebral veins in metastatic processes. Ann Intern Med. 1942;16:38-45.

27. Galasko CSB. Mechanisms of bone destruction in the development of skeletal metastases. Nature. 1976; 263(5577):507-508.

28. Togawa D, Lewandrowsky KU. The pathophysiology of spinal metastases. In: McLain RF, Lewandrowski KU, Markman M, Bukowski RM, Macklis R, Benzel EC, eds. Cancer in the Spine. Current Clinical Oncology. Totowa, NJ: Humana Press; 2006:17-23.

29. Musat E, Stefanescu C, Rusu V. Whole-body bone scintigraphy in the diagnosis and follow-up of the evolution of breast cancer. Rev Med Chir Soc Med Nat. 1999;103(1-2):163169.

30. Roth ES, Fetzer DT, Barron BJ, et al. Does colon cancer ever metastasize to bone first? A temporal analysis of colorectal cancer progression. BMC Cancer. 2009;9:274. doi:10.1186/ 1471-2407-9-274

31. Zhang L, Gong Z. Clinical characteristics and prognostic factors in bone metastases from lung cancer. Med Sci Monit. 2017;23:4087-4094. doi:10.12659/msm.902971

32. Wang C, Shen Y. Study on the distribution features of bone metastases in prostate cancer. Nucl Med Commun. 2012;33(4):379-383. doi:10.1097/MNM.0b013e3283504528
Disclosures and COI: The author declares that he has no conflict of interest. This research did not receive any specific grant from funding agencies in the public, commercial, or not-for-profit sectors. The author did not receive any outside funding, honorarium, grants, or other form of payment in support of his research for or preparation of this work.

Corresponding Author: Koray Başdelioğlu, MD, Istanbul Oncology Hospital Department of Orthopaedic and Traumatology, Cevizli Mah. Toros Cad. No: 86 Maltepe/Istanbul, Turkey. Phone : +90 55340453 37; Fax: +90 21645738 00; Email: drkoraybasd@gmail.com.

Published 26 February 2021

This manuscript is generously published free of charge by ISASS, the International Society for the Advancement of Spine Surgery. Copyright (C) 2021 ISASS. To see more or order reprints or permissions, see http://ijssurgery.com. 